\title{
Effects of Electronic Devices on Vision in Students Age Group 18-25
}

\author{
Mahar Safdar Ali Qasim¹, Maher Mustansar Ali Qasim², Memona Batool Qasim³ ${ }^{3}$ Rashid Asghar Khan ${ }^{4}$ \\ Naveed Anwar ${ }^{5 *}$, Saba Akram ${ }^{6}$ and Kehkshan Khalid ${ }^{7}$
}

${ }^{1}$ Department of Ophthalmology, Optometry \& Orthoptics at Riphah International University Lahore, Lahore, Pakistan; ${ }^{2}$ Department of Ophthalmology, Isra University Islamabad, Islamabad, Pakistan; ${ }^{3}$ Department of Ophthalmology, Women University, Multan, Pakistan; ${ }^{4}$ Department of Vision Sciences, University of Lahore, Lahore, Pakistan; ${ }^{5}$ Department of Rehab Sciences, Riphah International University, Lahore, Pakistan; ${ }^{6}$ Department of Optometry; University of Lahore, Lahore, Pakistan; ${ }^{7}$ Department of Sports physical therapy, Riphah International University, Lahore, Pakistan

Corresponding author: Naveed Anwar, Department of Rehab Sciences, Riphah International University, Lahore, Pakistan, E-mail: naveed.anwer@riphah.edu.pk

\section{Abstract}

Purpose: To increase understanding about the damaging effects of light rays on eyesight. To investigate frequency of people affected from light rays electronic devices. Study design: Cross sectional study. Place and Duration of Study: Riphah International University Lahore and duration of study was six months from June 2020 to December 2020. Materials \& Methods: A six months cross sectional study with convenient sampling method was conducted at Riphah international university Lahore from June 2020 to December 2020. The age of the individuals was between 18 years-25 years. Individuals who fulfill the inclusion criteria were chosen from University. A proforma was filled regarding detailed history of use of electronic devices other visual problems associated with it. Results: In present study 200 students were participated who has been using electronic devices. In this 100 students were male and 100 were females. $60 \%$ were emmetropic, $27.5 \%$ were myopic, $5.5 \%$ were hyperopia and $7.0 \%$ were astigmatic. $40 \%$ individuals were using smartphones from 11 years- 13 years, 20.5\% using from 8 years- 11 years and $25 \%$ using from 4 years- 7 years. The purpose of using these electronic devices in 96 individuals was using smartphone only for social networking. Most of them (130) participants were using smartphone and laptops. Usage of these electronic devices for more than 4 hours-6 hours was observed in 60 members. Among these individuals most of them were using these electronic devices by lying on the bed and some were also using in cervical flexion position. Conclusion: The over all research identified the smartphone/any electronic devices used so longer had bad effect on vision most particularly myopia occurred. This research suggests that constant and extreme utilization of smartphone and other electronic devices should be avoided.

Keywords: Electronic devices; Blue light; Usage of electronic devices

\section{Introduction}

Light can be divided into its basic components of colors e.g. red, orange, yellow, green, blue, indigo and violet. When merged, it becomes the white light that we see. Each of these components has a different energy and wavelength. Red end of spectrum has longer wavelengths and less energy. While on the other side, blue end has smaller wavelengths and high energy. Visible spectrum of light that appears white can have a huge blue part, which can endanger the eyesight to a greater extent of wavelength from the blue end of the scale. ${ }^{[1]}$

The largest supplier of blue light is sunshine. In addition to this, there are many other sources too e.g.

- $\quad$ Fluorescent light.

- CFL (Compact Fluorescent Light) bulbs.

- $\quad$ LED (Light Emitting Diode) light.

- $\quad$ Flat screen LED televisions.

- Computer monitors, smart phones, and tablet screens.

Exposure of the eyesight through light rays is small as compared to the extent of contact from the sun. But there is worry over the lasting effects of screen exposure because of the closeness of the 1572 screens and the duration of time that is used for looking at them. As current NEI-funded report, children's eyes take in more blue light than adults from electronic device screens. ${ }^{[2]}$

Almost all visible blue spectrums of light goes through the cornea and lens and focus at the retina. This light may disturb the eyesight and also affects the age of the eyes. Recent studies reveal that excessively exposure to blue end of light could lead to. ${ }^{[3]}$

\section{Digital eyestrain/Asthenopia}

Light rays from laptop screens and digital electronic devices can reduce contrast heading to digital eyestrain/asthenopia. Asthenopia is a condition in which pain in eyes, heaviness of eyes, dryness and headache are common. Sitting in front of digital devices screens, dryness of eyes, bad lightening or low brightness level can also cause eyestrain. Common symptoms of eyestrain involve sore or annoyed eyes and difficulty in focusing. ${ }^{[4]}$

This is an open access article distributed under the terms of the Creative Commons Attribution-NonCommercial-ShareAlike 3.0 License, which allows others to remix, tweak, and build upon the work non-commercially, as long as the author is credited and the new creations are licensed under the identical terms. 


\section{Retina damage}

Studies also suggest that prolonged exposure to light rays with time could also lead to damage in retinal cells. This may cause vision pathologies like age-related macular degeneration. ${ }^{[1]}$

If continuous contact to light rays from digital devices, laptop screens, tablets and smart phones is an issue, then there are following few ways to minimize the exposure of eye to blue light. ${ }^{[5]}$

\section{Screen time}

Try to reduce the extent of time wasted in front of these screens.

\section{Filters}

Many types of screen filters are available for smart phones, laptop screens and tablets. They minimize the quantity of blue light that reaches at the retina in eyes. ${ }^{[6]}$

\section{Computer glasses}

Computer spectacles with yellow-tinted lenses that prevent the blue light from entering the eyes can also reduce the eye strain. ${ }^{[7]}$

\section{Anti-reflective lenses}

Anti-reflective lenses reduce glare and improve contrast and also block blue light rays from the sun and digital electronic devices. ${ }^{[8]}$

The development in digital/electronic screen technology has improved significantly over the years, and many electronic digital devices use LED back-light modality to help improve screen illumination and clarity. These LEDs produce very intense blue light waves. Laptops, smartphones, flat screen television and tablets are the example of the devices that use this expertise. Eyes are being exposed to the abundant blue light by the widely use and increasing popularity of these electronic devices for the longer period of time. ${ }^{[3]}$

\section{Methodology}

Six months cross sectional study with convenient sampling method was conducted at Riphah International University Lahore from June 2020 to December 2020. The age of the individuals was between 18 years- 25 years. Individuals who fulfill the inclusion criteria were chosen from University. A proforma was filled regarding detailed history of use of electronic devices other visual problems associated with it. Inclusion criteria include the individuals who were using digital electronic devices and were willing to participate in this study. Exclusion criteria include the individuals who were not using digital electronic devices and were not willing to participate in this study.Permission was taken from research cell of Riphah International University Lahore and consent was taken from every selected student for the study. Data was collected by filling the proforma. Data entered and analyzed by using Statical Package of Social Scientist (SPSS) version 20.0. Variables were presented as frequency and percentage.

\section{Results}

The clinical characteristics are listed in Graphs 1-6. In present study 200 students were participated who has been using electronic devices. In this 100 students were male and 100 were females. While focusing the refractive error in Riphah International University's students, there were 55 Myopic, 11 hypermetropic and 14 astigmatic students. Frequency Graph number 1 show the distribution of refractive errors in students. There is no statistically correlation of refractive errors and age of the students [Figures 1-2].

About 40\% students were using electronic devices for 11 years-13 years. Frequency Graph number 2 shows distribution of years of usage of these electronic devices. In these students common posture for using these electronic devices was lying on the bed (36\%) and craning neck was $14.5 \%$. About $90 \%$ of students were using these electronic devices for social media streaming. Most of them (130) participants were using smartphone and laptops. Usage of these electronic devices for

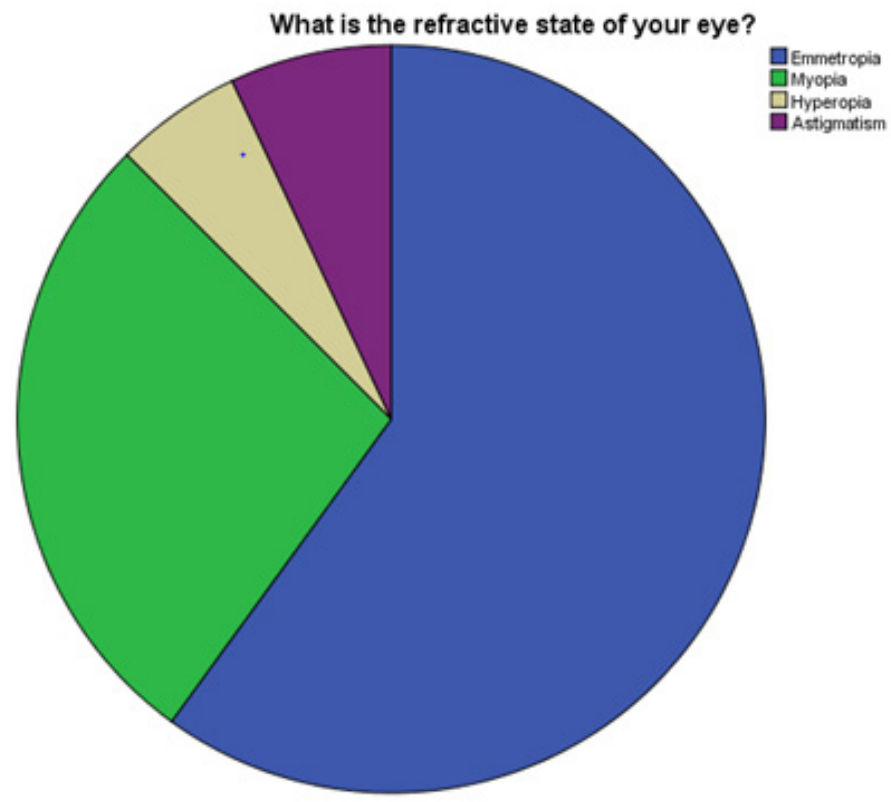

Graph 1: Refractive error in Riphah international university Lahore's students, there were 55 myopic students, 11 hyperopic, 14 astigmatic and 120 students were emmetropic.

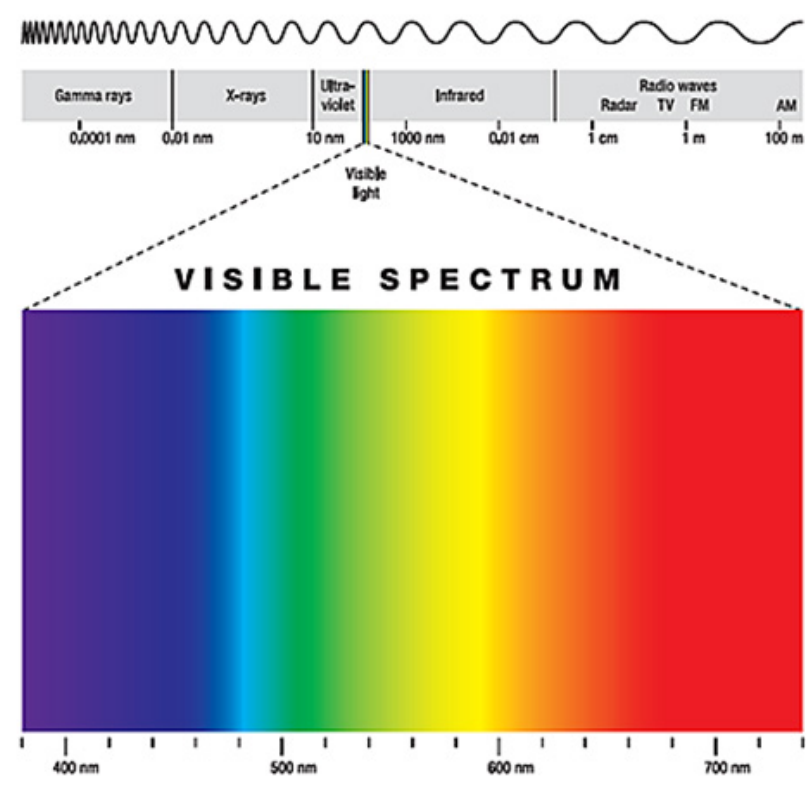

Figure 1: Visible spectrum wavelength. 
more than 4 hours- 6 hours were observed in 60 members.

\section{Discussion}

This research includes 200 samples from Riphah International University Lahore, which includes 100 males and 100 females. The closed ended questions were asked from students by the

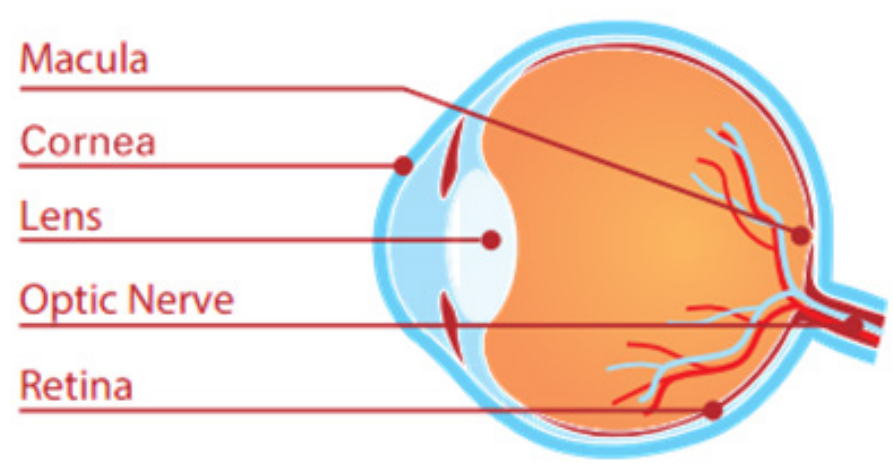

Figure 2: Blue spectrum of light goes through the cornea and lens and focus at the retina. aid of proforma, after taking consent from them. Most of the students were in the age of 18 years- 21 years and some were between the ages of 22 years- 25 years. The purpose of using electronic devices were social networking $40.5 \%$, playing games $7.5 \%$, watching dramas and movies $33.0 \%$, internet surfing $9.5 \%$ and for study purposes $9.5 \%$.

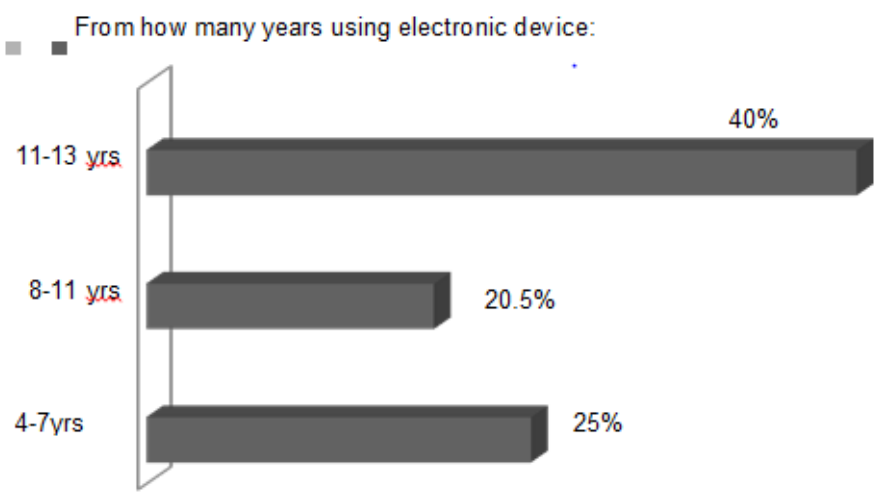

Graph 2: History of smartphone usage $14.5 \%$ was using electronic gadgets from 15 years.
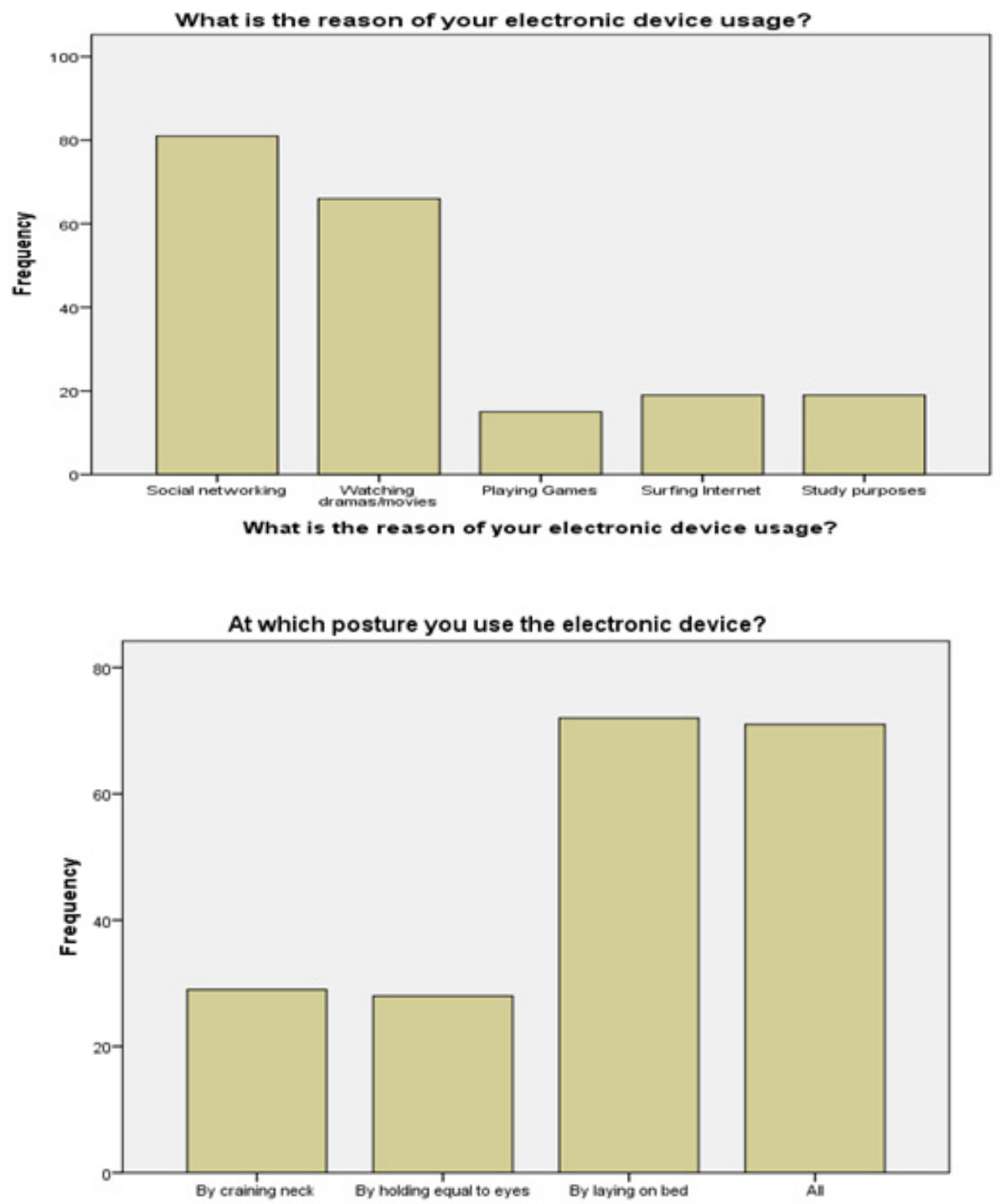

At which posture you use the electronic device?

Graph 3: Type of devices used by students. 


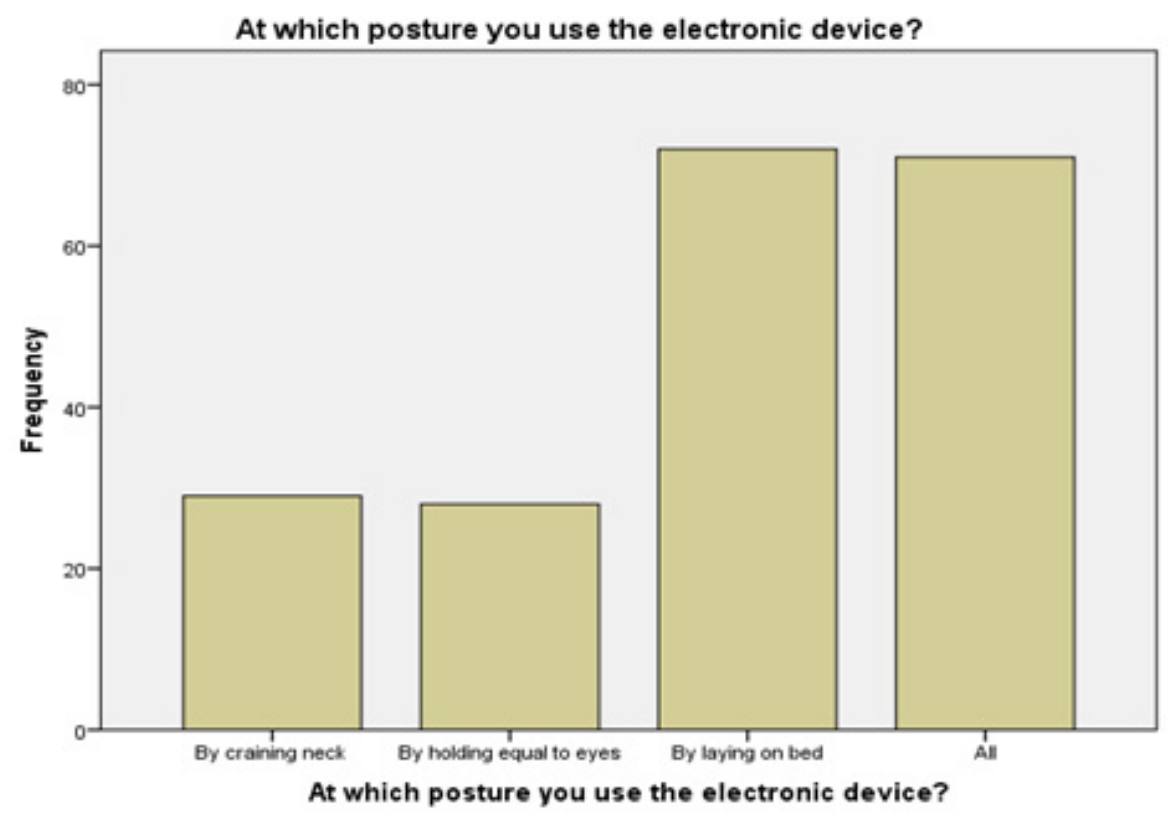

Graph 4: The posture of using devices. The most common posture was by usage of electronic device by laying on bed $36.0 \%$, by holding equal to eyes $14.0 \%$, by craning neck $14.5 \%$ and all $35.5 \%$.

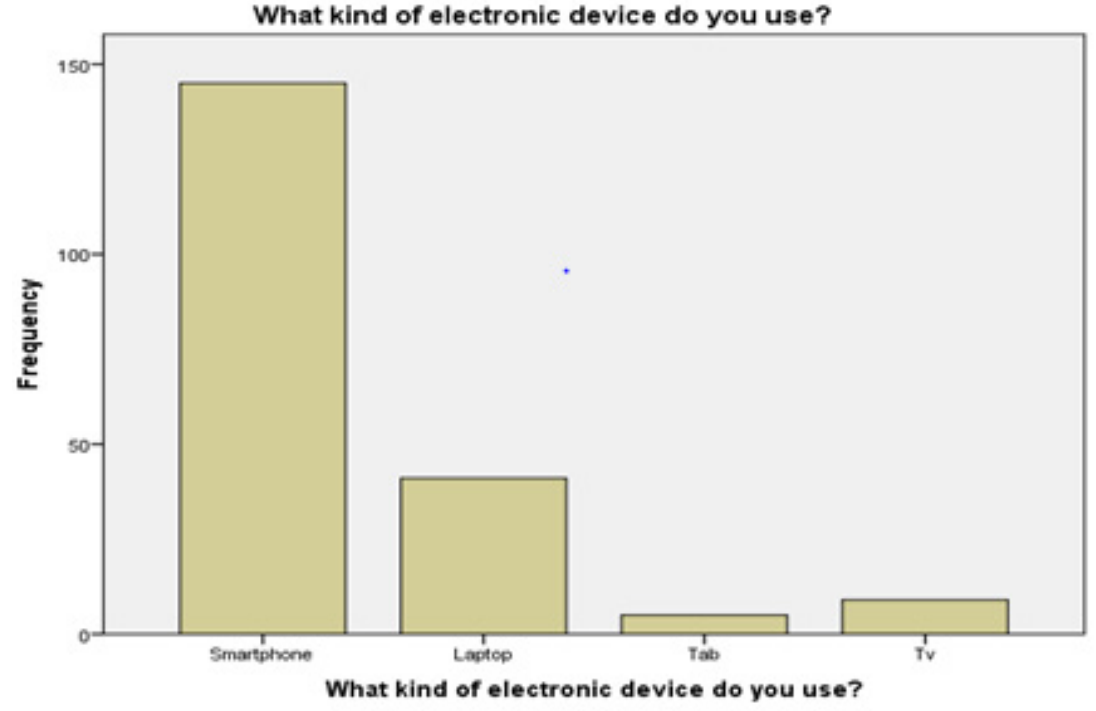

Graph 5: Purpose of using electronic devices.

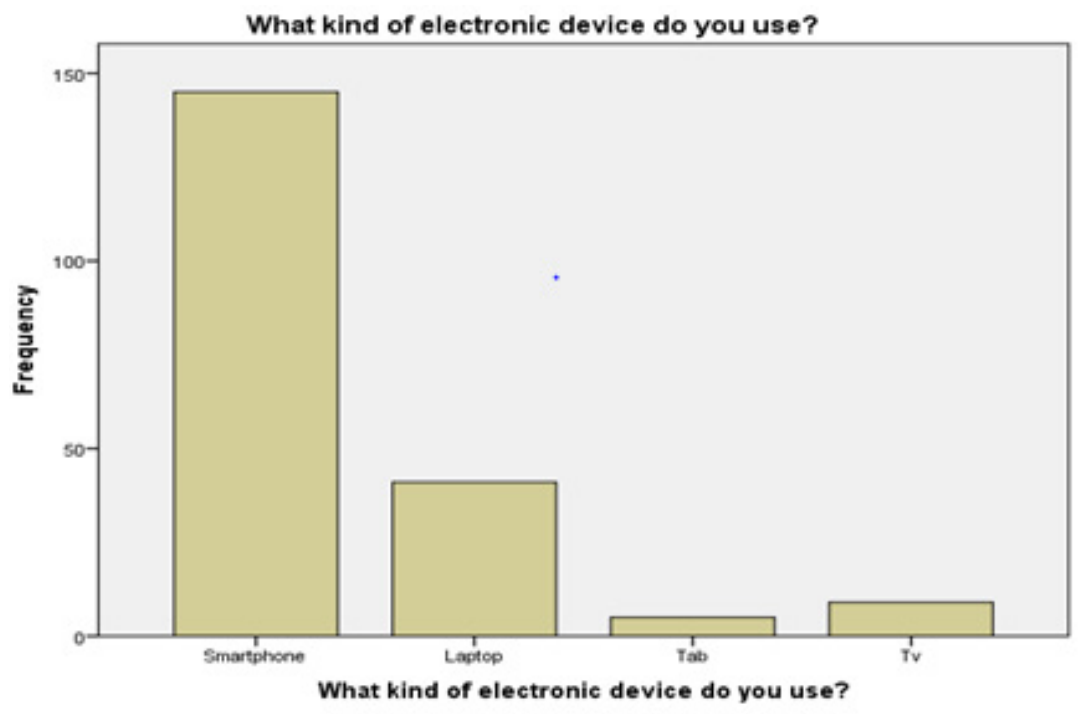

Graph 6: Time spending daily on electronic device. 
Mostly students prefer medium font size. Vision is more working while seeing at near and small screens, light rays emit from the phone have great effect on human eyes when we use smartphone people face blurred vision. And most of the students wear their prescription while using smartphone. Many students had complained of headache after a continuous long use of smartphone. More than $80 \%$ of students use smartphone in darkness by lying on bed. At this position the distance of smartphone screen from become less than $18 \mathrm{~cm}$, which is a big risk factor of development of myopia, that's why now a day's smartphones are the major cause of myopia in people who are using smartphone for a prolonged period of time. It is important to know that the different levels of brightness also has great effect on human eye like when you use low brightness in sunlight or in brighter area your ayes need more accommodation which cause eye strain same in case if you use high brightness in dark it also cause fatigue and headache. Barar et al. the excessive usage of these electronic digital devices points a new challenge of digital asthenia and eyestrain. This study shows the electronic devices usage by the young children. In addition to the use of these devices, take-home assignments to the participants, one-third of the studies reported with the use of digital reading devices, instead of a regular textbook. The majority of the students prefer to sit in a chair while you read ( $77 \%$, the 445 students), and only $21 \%$ ( 123 of the students would prefer to lie down on the bed, and 8 , students alternated between the chairs, and the beds are. devices. The frequencies of our study show same results as in this study. ${ }^{[9-13]}$ Rofenfiled et al. study shows the same results as in our study. In that study the $55 \%$ students were using electronic devices by lying on the bed and $20 \%$ by craning the neck posture. ${ }^{[10-15]}$ Costana et al. in his study titled visual and ocular symptoms related to the use of video display terminals which was published in journal of behavioral optometry showed that the students suffering from eyestrain by using smart phone was $20 \%$. 50\% students used their electronic devices by lying on their bed and they also suffered from asthenopia. ${ }^{[16-22]}$ This study also showed the same frequencies as in the above mentioned study.In another study which was conducted by the Sheedyet al. titled asthenopia by using electronic devices showed that there were $40 \%$ students who used electronic devices by lying on the bed position and $20 \%$ were suffered by the eyestrain. This study also showed the same frequencies. ${ }^{[22-25]} \mathrm{A}$ study titled children and digital technologies which was conducted by the Daum et al. showed that there were $55 \%$ students who were using their electronic devices by lying on the bed and $22 \%$ suffered by the eyestrain. These results also strengthen our study and the frequencies are almost same in both studies. Blue light emitting from the screen of smartphone causes disturbed sleep cycle, and when an electronic device is used in darkness it has more harmful effects. The whole research discussion tells that smartphone's excessive use causes many visual problem most particularly myopia in refractive errors and in ocular symptoms, headache was the most common problem. ${ }^{[25]}$

\section{Conclusion}

The overall research identified the smartphone/any electronic devices used so longer had bad effect on vision most particularly myopia occurred. While the use of smartphones is increased in last 5 years-10 years and myopia in Riphah International University Lahore students was $27.5 \%$, hyperopia was $5.5 \%$, astigmatism was $7.0 \%$. This research suggests that continuous excessive use of smartphone and other electronic gadgets should be avoided.

\section{Recommendations}

Students should be aware of bad effect of prolonged use of video display gadgets on eyes. If possible students should not use computer/mobile more than 4 hours continuously in a day. And if it is necessary to use computer/mobile for prolonged period in a day then preventive measure must be applied continuously. If students have other ocular problems such as myopia, continuous use of display gadgets should be avoided. Don't use at low brightness in excessive light such as sunlight and at high brightness in darkness. Use an Anti-blue tempered glass protector on phone. Anti-blue coating glass is a unique glass shield which contains anti-blue ray. There is a layer of thin film which can stop the blue ray coming from the LED/LCD screen, so it block the emitting, and prevent it from reaching at the retina of the eyes. It reduces the mobile LCD glare. Take breaks after every 20 minutes, close the eyes for 20 seconds and look forward for 20 seconds (Use 20-20-20 rule).

\section{References}

1. Barar A, Pellanda LC, Fassa AG, Castagno VD. Prevalence of asthenopia in children: A systematic review with meta-analysis. J Pediatr. 2015;91:320-325.

2. Vilela MA, Castagno VD, Meucci RD, Fassa AG. Asthenopia in schoolchildren. Clin Ophthalmol. 2015;9:1595-1603.

3. Long J, Cheung R, Duong S, Paynter R, Asper L. Viewing distance and eyestrain symptoms with prolonged viewing of smartphones. Clin Exp Optom. 2017;100:133-137.

4. Silva GR, Pitangui AC, Xavier MK, Correia-Junior MA, De Araujo RC. Prevalence of musculoskeletal pain in adolescents and association with computer and videogame use. J Pediatr. 2016;92:188-196.

5. Husarova D, Geckova AM, Blinka L, Sevcikova A, van Dijk JP, Reijneveld SA. Screen-based behaviour in school-aged children with long-term illness. BMC Public Health. 2016;16:130-135.

6. Joo J, Sang Y. Exploring Koreans' smartphone usage: An integrated model of the technology acceptance model and uses and gratifications theory. Comput Hum Behav. 2013;29:2512-2518.

7. Drummond A, Sauer JD. Video-games do not negatively impact adolescent academic performance in science, mathematics or Reading. PLoS One. 2014;9:20-30.

8. Tosini G, Ferguson I, Tsubota K. Effects of blue light on the circadian system and eye physiology. Mol Vis. 2016;22:61-67.

9. Yoshimura M, Kitazawa M, Maeda Y, Mimura M, Tsubota K, Kishimoto T. Smartphone viewing distance and sleep: An experimental study utilizing motion capture technology. Nat Sci Sleep. 2017;9:59-63.

10. Wolffsohn JS, Arita R, Chalmers R. TFOS DEWS II diagnostic methodology report. Ocul Surf 2017;15:539-574.

11. Moon JH, Lee MY, Moon NJ. Association between video display terminal use and dry eye disease in school children. J Pediat Ophth Strab. 2014;51:87-92. 
12. Moon JH, Kim KW, Moon NJ. Smartphone use is a risk factor for pediatric dry eye disease according to region and age: A case control study. BMC Ophthalmol. 2016;16:188-190

13. Courtin R, Pereira B, Naughton G. Prevalence of dry eye disease in visual display terminal workers: A systematic review and metaanalysis. BMJ Open. 2016;6:1-10.

14. Tauste A, Ronda E, Molina MJ. Effect of contact lens use on computer vision syndrome. Ophthalmic Physiol Opt. 2016;36:112-119.

15. Seguí MM,CabreroGJ, Crespo A. A reliable and valid questionnaire was developed to measure computer vision syndrome at the workplace. J Clin Epidemiol. 2015;68:662-673.

16. Vilela MAP, Pellanda LC, Fassa AG. Prevalence of asthenopia in children: A systematic review with meta-analysis. J Pediatr. 2015;91:320-325.

17. Benedetto S, Drai-Zerbib V, Pedrotti M. E-readers and visual fatigue. PLoS One. 2013;8:1-8.

18. Lin JB, Gerratt BW, Bassi CJ. Short-wavelength light-blocking eyeglasses attenuate symptoms of eye fatigue. Investig Ophthalmol
Vis Sci. 2017;58:442-447.

19. Ide T, Toda I, Miki E. Effect of blue light-reducing eye glasses on critical flicker frequency. Asia Pac J Ophthalmol. 2015;4:80-85.

20. Rosenfield M, Jahan S, Nunez K. Cognitive demand, digital screens and blink rate. Comput Human Behav. 2015;51:403-406.

21. Bhargava R, Kumar P, Phogat H. Oral omega-3 fatty acids treatment in computer vision syndrome related dry eye. Cont Lens Anterior. 2015;38:206-210.

22. Portello JK, Rosenfield M, Chu CA. Blink rate, incomplete blinks and computer vision syndrome. Optom Vis Sci. 2013;90:482-487.

23. Long J, Cheung R, Duong S. Viewing distance and eyestrain symptoms with prolonged viewing of smartphones. Clin Exp Optom. 2017;100:133-137.

24. Tosini G, Ferguson I, Tsubota K. Effects of blue light on the circadian system and eye physiology. Mol Vis. 2016;22:61-72

25. O'Hagan JB, Khazova M, Price LLA. Low-energy light bulbs, computers, tablets and the blue light hazard. Eye. 2016;30:230-233. 